\title{
KREĨN SPACE UNITARY DILATIONS OF HILBERT SPACE HOLOMORPHIC SEMIGROUPS
}

\author{
STEFANIA A. M. MARCANTOGNINI
}

\begin{abstract}
The infinitesimal generator $A$ of a strongly continuous semigroup on a Hilbert space is assumed to satisfy that $B_{\beta}:=A-\beta$ is a sectorial operator of angle less than $\frac{\pi}{2}$ for some $\beta \geq 0$. If $B_{\beta}$ is dissipative in some equivalent scalar product then the Naimark-Arocena representation theorem is applied to obtain a Kreĭn space unitary dilation of the semigroup.
\end{abstract}

\section{INTRODUCTION}

A Kreın space unitary dilation of a strongly continuous semigroup $\{T(t)\}$ on a Hilbert space $\mathfrak{H}$ was built up by B. McEnnis under the assumption that the numerical range of the infinitesimal generator $A$ lies on a sector of semi-angle $0<\theta<\frac{\pi}{2}$ around the real line [10]. McEnnis's construction follows the one given by C. Davis for uniformly continuous semigroups [5]. Both constructions lean on the existence of a selfadjoint operator $G$ such that

$$
\frac{d}{d t}\|T(t) h\|^{2}=\langle G T(t) h, T(t) h\rangle, \quad \frac{d}{d t}\left\|T(t)^{*} h\right\|^{2}=\left\langle G T(t)^{*} h, T(t)^{*} h\right\rangle
$$

for all $t>0$ and all $h \in \mathfrak{H}$. The key element in McEnnis's construction is the convexity of the numerical range (the Hausdorff-Toeplitz theorem), which grants the $m$ - $\theta$-dissipativeness of $A-\beta$ for some $\beta \geq 0$ and, in consequence, a contractive holomorphic extension of $\left\{e^{-\beta t} T(t)\right\}$ within the sector $|\arg (z)|<\frac{\pi}{2}-\theta$.

The sectoriality of the numerical range can be replaced by sectoriality of the spectrum together with some norm estimates of the resolvent. The latter conditions allow a functional calculus which, in turn, gives a one-to-one correspondence between the type of closed operators $A$ satisfying those constraints and the bounded holomorphic strongly continuous semigroups $\{T(z)\}$ (see, for instance, [8]). On the other hand, if the calculus is $H^{\infty}$-bounded then the operator $A$ is dissipative in some equivalent Hilbert space inner product (refer to [8] for details). In sum, McEnnis's result can be achieved under the weaker conditions that combine sectoriality

2010 Mathematics Subject Classification. Primary: 47D03; Secondary: 47B44.

Key words and phrases. Hilbert space holomorphic semigroups; KreĬn space unitary groups; sectorial operators; Naimark's representation theorem.

The work was done during my sojourn in the Instituto Argentino de Matemática in a position funded by CONICET. I am deeply grateful to the Instituto for the hospitality and to CONICET for the financial support. 
(of the spectrum) with bounded $H^{\infty}$-calculus. We apply the Naimark-Arocena representation theorem to obtain the Krĕn space unitary dilation, though. So this note serves as a slightly more general result than the one by McEnnis, as well as an alternate of its proof.

This note is organized as follows: Section 2 includes some preliminaries, while Section 3 presents the results.

\section{PRELIMINARIES}

In the sequel we assume that all Hilbert spaces are complex and separable.

Given a Hilbert space $(\mathfrak{H},\langle\cdot, \cdot\rangle)$, we denote by $\mathfrak{L}(\mathfrak{H})$ the linear space of all bounded linear operators on $\mathfrak{H}$. When $A$ is a linear operator which is not everywhere defined on $\mathfrak{H}$, we write $\mathcal{D}(A)$ for its domain. The symbol $\mathcal{R}(A)$ stands for the range of $A$. The numerical range of $A$ is the set

$$
\nu(A):=\{\langle A x, x\rangle: x \in \mathcal{D}(A),\|x\|=1\} .
$$

If $A: \mathcal{D}(A) \rightarrow \mathfrak{H}$ is a closed linear operator, then

$$
\begin{aligned}
\rho(A) & :=\left\{\lambda \in \mathbb{C}:(\lambda-A)^{-1} \in \mathfrak{L}(\mathfrak{H})\right\}, \\
\sigma(A) & :=\mathbb{C} \backslash \rho(A), \quad \text { and } \\
R(\lambda, A) & :=(\lambda-A)^{-1} \quad(\lambda \in \rho(A))
\end{aligned}
$$

are its resolvent set, spectrum, and resolvent, respectively.

2.1. Holomorphic semigroups. References on semigroup theory abound; 11] is amongst the classical textbooks on the subject. We recall that a strongly continuous (one-parameter) semigroup on a Hilbert space $(\mathfrak{H},\langle\cdot, \cdot\rangle)$ is a family $\{T(t)\} \subseteq \mathfrak{L}(\mathfrak{H})$ parameterized by $t \geq 0$ that satisfies the following conditions:

(i) $T(0)=1$ and $T(s+t)=T(s) T(t)$ for all $s, t \geq 0$.

(ii) $T(t)$ converges strongly to 1 as $t \rightarrow 0^{+}$.

When $\|T(t)\| \leq 1$ for all $t \geq 0,\{T(t)\}$ is said to be a contraction semigroup.

The infinitesimal generator $A$ of a strongly continuous semigroup $\{T(t)\}$ is defined as $A x:=\lim _{t \rightarrow 0^{+}} t^{-1}(T(t)-1) x$, the domain $\mathcal{D}(A)$ of $A$ being the set of those $x \in \mathfrak{H}$ for which the limit exists. $A$ is known to be a densely defined closed operator.

We also recall that a linear operator $A$ on a Hilbert space $\mathfrak{H}$ with domain $\mathcal{D}(A)$ is said to be dissipative if $\operatorname{Re}\langle A x, x\rangle \leq 0$ for all $x \in \mathcal{D}(A)$, that is, if $\nu(A)$ is contained in the closed left half-plane. A dissipative operator $A$ is called $m$-dissipative if $A$ is closed and $\mathcal{R}(1-A)=\mathfrak{H}$.

Proposition 2.1. The following assertions are equivalent:

(a) A is m-dissipative.

(b) $A$ is a densely defined closed operator and $A^{*}$ is $m$-dissipative.

(c) $A$ is a densely defined closed operator, $\sigma(A) \subseteq\{\lambda \in \mathbb{C}: \operatorname{Re} \lambda \leq 0\}$, and $\|R(\lambda, A)\| \leq(\operatorname{Re} \lambda)^{-1}$ for all $\operatorname{Re} \lambda>0$.

(d) A generates a strongly continuous contraction semigroup. 
(a) $\Leftrightarrow(\mathrm{d})$ and (c) $\Leftrightarrow$ (d) are the renowned Lumer-Phillips theorem and HilleYosida theorem, respectively.

Let $0<\omega \leq \frac{\pi}{2}$. An $\mathfrak{L}(\mathfrak{H})$-valued function $T$ on the additive semigroup

$$
X(\omega):=\{z \in \mathbb{C}: z \neq 0,|\arg (z)|<\omega\} \cup\{0\}
$$

is called a strongly continuous holomorphic semigroup if:

(i) $z \mapsto T(z)$ is holomorphic in $X(\omega)$.

(ii) $T(0)=1$ and $T\left(z+z^{\prime}\right)=T(z) T\left(z^{\prime}\right)$ for all $z, z^{\prime} \in X(\omega)$.

(iii) For each $0<\phi<\omega, T(z)$ converges strongly to 1 as $z \rightarrow 0$ within $X(\phi)$.

The holomorphic semigroup $\{T(z)\}$ on $X(\omega)$ is said to be bounded when:

(iv) $\sup \{\|T(z)\|: z \in X(\phi)\}<\infty$ for each $0<\phi<\omega$.

Above and in the following, $\arg (z)$ is to be set in the interval $(-\pi, \pi]$ for $z \in$ $\mathbb{C} \backslash\{0\}$.

Let $0<\theta<\frac{\pi}{2}$. A linear operator $A$ on $\mathfrak{H}$ with domain $\mathcal{D}(A)$ is said to be $\theta$-dissipative if $\nu(A)$ lies in the sector

$$
S_{0, \theta}:=\{\lambda \in \mathbb{C}: \lambda \neq 0,|\arg (\lambda)| \geq \pi-\theta\} \cup\{0\} .
$$

A $\theta$-dissipative operator $A$ is $m$ - $\theta$-dissipative if $A$ is $m$-dissipative.

Proposition 2.2. The following assertions are equivalent:

(a) $A$ is $m$ - $\theta$-dissipative.

(b) A generates a strongly continuous holomorphic semigroup $\{T(z)\}$ on $X\left(\frac{\pi}{2}-\theta\right)$ satisfying $\|T(z)\| \leq 1$ for all $z \in X\left(\frac{\pi}{2}-\theta\right)$.

We point out that $A$ is said to be $(\theta-)$ accretive if $-A$ is $(\theta-)$ dissipative. The $(m-) \theta$-accretive operators were extensively studied by T. Kato 9 . He called them $(m-)$ sectorial operators but nowadays the term "sectorial" refers to a different type of operator. In the following we will call $A$ a sectorial operator if $\sigma(A) \subseteq S_{0, \theta}$ for some $0<\theta<\pi$ and, for each $\theta<\phi<\pi, \sup \left\{\|\lambda R(\lambda, A)\|: \lambda \in \mathbb{C} \backslash S_{0, \phi}\right\}<\infty$. We will restrict ourselves to sectorial operators of angle $0<\theta<\frac{\pi}{2}$. A comprehensive account of sectorial operators is [8]. Therein the definition of sectorial operator is different from the one we adopt, though. Roughly, our definiton corresponds with $-A$ being sectorial in the above mentioned monograph.

The most famous result on the numerical range is the Hausdorff-Toeplitz theorem which asserts that the numerical range of any (perhaps unbounded and not densely defined) linear operator on a complex or real (pre-)Hilbert space is convex. The convexity of the numerical range is one of the key elements in showing the following result from [9] (cf. [10]).

Proposition 2.3. Let $A$ be the infinitesimal generator of a strongly continuous semigroup $\{T(t)\} \subseteq \mathfrak{L}(\mathfrak{H})$. If $\nu(A)$ lies in the sector

$$
S_{\alpha, \theta}:=\{\lambda \in \mathbb{C}: \lambda \neq \alpha,|\arg (\lambda-\alpha)| \geq \pi-\theta\} \cup\{\alpha\}
$$

of vertex $\alpha \geq 0$ and semi-angle $0<\theta<\frac{\pi}{2}$, then there exists $\beta \geq 0$ such that $A-\beta$ is $m$ - $\theta$-dissipative. 
By combining Proposition 2.3 with Proposition 2.2 we get that, under the assumption that

$$
\nu(A) \subseteq S_{\alpha, \theta} \quad(\alpha \geq 0,0<\theta<\pi / 2),
$$

there exists $\beta \geq 0$ such that $\left\{e^{-\beta z} T(z)\right\}$ is a contraction holomorphic semigroup on $X\left(\frac{\pi}{2}-\theta\right)$. Hence, if we consider only real $t$, we obtain that

$$
\|T(t)\| \leq e^{\beta t} \quad \text { for all } t \geq 0
$$

and

$$
A^{n} T(t)=\frac{d}{d t^{n}} T(t) \quad \text { for every } t>0 \text { and } n \in \mathbb{N} .
$$

Holomorphic semigroups are somehow in between the general class of strongly continuous semigroups and the particular class of uniformly continuous semigroups (for which the infinitesimal generator is bounded). The functional calculus for sectorial operators gives a one-to-one correspondence between sectorial operators $A$ with $0 \leq \theta<\frac{\pi}{2}$ and bounded holomorphic strongly continuous semigroups $\{T(z)\}$ on $X\left(\frac{\pi}{2}-\theta\right)$ (see [8]). Therefore 2.3) is granted under the weaker condition of sectoriality. On the other hand, $(2.2)$ holds if $A-\beta$ is $m$-dissipative.

We replace (2.1) by the following hypothesis: there exists $\beta \geq 0$ such that $A-\beta$ is sectorial of angle $0<\theta<\frac{\pi}{2}$ and $\operatorname{Re}\langle A x, x\rangle_{0} \leq \beta\|x\|_{0}^{2}$ for all $x \in \mathcal{D}(A)$, with $\langle\cdot, \cdot\rangle_{0}$ an equivalent scalar product on $\mathfrak{H}$. This happens, for instance, if $A-\beta$ is sectorial and has bounded $H^{\infty}$-calculus with $H^{\infty}$-angle less than $\frac{\pi}{2}$ (the reader is referred to 8 for the definitions). We remark that it is not always the case that a sectorial operator $A$ on a Hilbert space, with sectorial angle less than $\frac{\pi}{2}$, has bounded $H^{\infty}$-calculus.

2.2. Krĕn spaces. As familiarity with operator theory on Kreı̆n spaces is presumed, only some notation is introduced. We emphasize that the common Hilbert space notation is carried over into the Kreŭn space setting.

Given a fundamental decomposition $\mathfrak{K}=\mathfrak{K}^{+} \oplus \mathfrak{K}^{-}$of the Kreln space $(\mathfrak{K},\langle\cdot, \cdot\rangle)$, we write $|\mathfrak{K}|$ for $\mathfrak{K}$ viewed as the Hilbert space relative to the fundamental decomposition. Therefore, if $J$ is the corresponding fundamental symmetry, that is, $J x=x^{+}-x^{-}$whenever $x=x^{+}+x^{-}$with $x^{ \pm} \in \mathfrak{K}^{ \pm}$, then $\langle x, y\rangle_{|\mathfrak{K}|}=\langle J x, y\rangle$ and $\langle x, y\rangle=\langle J x, y\rangle_{|\mathfrak{K}|}$ for all $x, y \in \mathfrak{K}$.

By $\mathfrak{L}(\mathfrak{K})$ we mean the space of all everywhere defined continuous linear operators on the Kreln space $\mathfrak{K}$. The space $\mathfrak{L}(\mathfrak{K})$ has the structure of a Banach space depending on the choice of a fundamental decomposition and the associated Hilbert space $|\mathfrak{K}|$. The corresponding operator norm for $\mathfrak{L}(\mathfrak{K})$ is the norm $\|\cdot\|$ of $\mathfrak{L}(|\mathfrak{K}|)$. We point up that any two operator norms for $\mathfrak{L}(\mathfrak{K})$ are equivalent and provide its topology. If $\mathfrak{K}$ and $\mathfrak{G}$ are two Krĕn spaces, $\mathfrak{L}(\mathfrak{K}, \mathfrak{G})$ and the operator norm are defined likewise.

For each $A \in \mathfrak{L}(\mathfrak{K}, \mathfrak{G})$ there is a unique $A^{*} \in \mathfrak{L}(\mathfrak{G}, \mathfrak{K})$ so that $\langle A x, y\rangle_{\mathfrak{G}}=$ $\left\langle x, A^{*} y\right\rangle_{\mathfrak{K}}$ for all $x \in \mathfrak{K}$ and $y \in \mathfrak{G}$.

We say that (i) $P \in \mathfrak{L}(\mathfrak{K})$ is a projection if $P^{2}=P=P^{*}$; (ii) $U \in \mathfrak{L}(\mathfrak{K})$ is a unitary operator if $U^{*} U=1=U U^{*}$. 
The regular subspaces of $\mathfrak{K}$ are those that are the ranges of projections. If $\mathfrak{F}$ is a regular subspace of $\mathfrak{K}$ we write $P_{\mathfrak{F}}$ to indicate the orthogonal projection from $\mathfrak{K}$ onto $\mathfrak{F}$.

Standard references on Kreı̆n spaces and operators on them are [1, 3, 4, We also refer to [6, 7] as authoritative accounts of the subject.

2.3. Naimark-Arocena representation theorem. The Naimark theorem characterizes those operator-valued Toeplitz kernels which have Hilbert space unitary representations. The theorem has an extension to the Krĕn space setting due to R. Arocena [2].

Theorem 2.4. Let $\Gamma$ be a group and denote by e its neutral element. Let $(\mathfrak{H},\langle\cdot, \cdot\rangle)$ be a Hilbert space. Let $f: \Gamma \rightarrow \mathfrak{L}(\mathfrak{H})$ be a Hermitian function such that $f(e)=1$. Assume that there exists a kernel $k: \Gamma \times \Gamma \rightarrow \mathfrak{L}(\mathfrak{H})$ with the following properties:

(i) $k(e, e)=1$.

(ii) $k$ is a majorant of $f$, in the sense that there exists $r>0$ such that

$$
\left|\sum_{s, t \in \Gamma}\left\langle f\left(t^{-1} s\right) h(s), h(t)\right\rangle\right| \leq r \sum_{s, t \in \Gamma}\langle k(s, t) h(s), h(t)\rangle
$$

for every function $h: \Gamma \rightarrow \mathfrak{H}$ with finite support.

(iii) There exists $R>0$ such that, for any $h: \Gamma \rightarrow \mathfrak{H}$ with finite support such that $\sum_{s, t \in \Gamma}\langle k(s, t) h(s), h(t)\rangle>0$, there exists another function $h^{\prime}: \Gamma \rightarrow \mathfrak{H}$ with finite support satisfying $\sum_{s, t \in \Gamma}\left\langle k(s, t) h^{\prime}(s), h^{\prime}(t)\right\rangle>0$ and

$$
\frac{\left|\sum_{s, t \in \Gamma}\left\langle f\left(t^{-1} s\right) h(s), h^{\prime}(t)\right\rangle\right|}{\left(\sum_{s, t \in \Gamma}\langle k(s, t) h(s), h(t)\rangle\right)^{\frac{1}{2}}\left(\sum_{s, t \in \Gamma}\left\langle k(s, t) h^{\prime}(s), h^{\prime}(t)\right\rangle\right)^{\frac{1}{2}}} \geq R .
$$

(iv) There exists a function $\rho: \Gamma \rightarrow(0, \infty)$ such that

$$
\sum_{s, t \in \Gamma}\langle k(\xi s, \xi t) h(s), h(t)\rangle \leq \rho(\xi) \sum_{s, t \in \Gamma}\langle k(s, t) h(s), h(t)\rangle
$$

for all $h: \Gamma \rightarrow \mathfrak{H}$ with finite support and all $\xi \in \Gamma$.

Then there exist a Kreŭn space $\left(\mathfrak{K},\langle\cdot, \cdot\rangle_{\mathfrak{K}}\right)$ containing $(\mathfrak{H},\langle\cdot, \cdot\rangle)$ as regular subspace and a unitary representation $U(s)$ of $\Gamma$ in $\mathfrak{K}$ such that:

1) $f(s)=\left.P_{\mathfrak{H}} U(s)\right|_{\mathfrak{H}}$ for all $s \in \Gamma$, and

2) $\mathfrak{K}=\bigvee\{U(s) \mathfrak{H}: s \in \Gamma\}$, that is, $\mathfrak{K}$ is the space generated by $U(s) \mathfrak{H}, s \in \Gamma$.

Conversely, if there exist a Krĕn space $\left(\mathfrak{K},\langle\cdot, \cdot\rangle_{\mathfrak{K}}\right)$ containing $(\mathfrak{H},\langle\cdot, \cdot\rangle)$ as regular subspace and a unitary representation $U(s)$ of $\Gamma$ in $\mathfrak{K}$ such that 1) and 2) hold, then there exists a kernel $k$ satisfying (i), (ii), (iii), and (iv). 


\section{THE DILATION}

Hereafter $\{T(t)\}$ is a strongly continuous semigroup of bounded linear operators on a Hilbert space $(\mathfrak{H},\langle\cdot, \cdot\rangle)$ with infinitesimal generator $A$.

Let $\langle\cdot, \cdot\rangle_{A}$ be the graph scalar product on $\mathcal{D}(A)$ :

$$
\langle x, y\rangle_{A}:=\langle x, y\rangle+\langle A x, A y\rangle \quad(x, y \in \mathcal{D}(A)) .
$$

By the Riesz-Fréchet representation theorem, there exists a bounded linear operator $R$ on $\left(\mathcal{D}(A),\langle\cdot, \cdot\rangle_{A}\right)$ such that

$$
\langle A x, y\rangle+\langle x, A y\rangle=\langle x, R y\rangle_{A}=\langle R x, y\rangle_{A} \quad \text { for all } x, y \in \mathcal{D}(A) .
$$

Since $A$ is a densely defined closed operator on $(\mathfrak{H},\langle\cdot, \cdot\rangle)$, the von Neumann theorem (see [12]) assures that $A^{*} A, A A^{*}$ are selfadjoint and $1+A^{*} A, 1+A A^{*}$ are invertible. Let $h \in \mathfrak{H}$ and write $h=\left(1+A^{*} A\right) y, y \in \mathcal{D}(A)$. For all $x \in \mathcal{D}(A)$,

$$
\begin{aligned}
\langle R x, h\rangle & =\left\langle R x,\left(1+A^{*} A\right) y\right\rangle=\left\langle\left(1+A^{*} A\right) R x, y\right\rangle=\langle R x, y\rangle_{A}=\langle x, R y\rangle_{A} \\
& =\left\langle x,\left(1+A^{*} A\right) R y\right\rangle=\left\langle x,\left(1+A^{*} A\right) R\left(1+A^{*} A\right)^{-1} h\right\rangle .
\end{aligned}
$$

If $(S, \mathcal{D}(S))$ is the operator on $(\mathfrak{H},\langle\cdot, \cdot\rangle)$ given by $\mathcal{D}(S):=\mathcal{D}(A)$ and $S x:=R x$, then it follows that $\mathcal{D}\left(S^{*}\right)=\mathfrak{H}$ and $S^{*}=\left(1+A^{*} A\right) S\left(1+A^{*} A\right)^{-1}$. Thus $\left(\left(1+A^{*} A\right) S\right)^{*} \supseteq$ $S^{*}\left(1+A^{*} A\right)=\left(1+A^{*} A\right) S$.

We then get that $H:=\left(1+A^{*} A\right) S$ is a symmetric operator on $(\mathfrak{H},\langle\cdot, \cdot\rangle)$ with $\mathcal{D}(H)=\mathcal{D}(A)$ such that

$$
\langle H x, y\rangle=\langle A x, y\rangle+\langle x, A y\rangle \quad \text { for all } x, y \in \mathcal{D}(A) .
$$

Lemma 3.1. If there exists $\beta \geq 0$ such that $A-\beta$ is sectorial of angle $0<\theta<\frac{\pi}{2}$ and dissipative then the symmetric operator $H$ in (3.1) satisfies

$$
\langle H x, x\rangle \leq 2 \beta\|x\|^{2} \quad \text { for all } x \in \mathcal{D}(A)
$$

and

$$
\frac{d}{d t}\|T(t) h\|^{2}=\langle H T(t) h, T(t) h\rangle \quad \text { for all } t>0 \text { and } h \in \mathfrak{H} .
$$

Proof. Since $B:=A-\beta$ is dissipative, it follows that, for all $x \in \mathcal{D}(A),\langle H x, x\rangle=$ $2 \operatorname{Re}\langle B x, x\rangle+2 \beta\|x\|^{2} \leq 2 \beta\|x\|^{2}$. On the other hand, $B$ is $m$-dissipative, for $B$ is sectorial of angle $0<\theta<\frac{\pi}{2}$ and dissipative. Therefore, $B$ generates a strongly continuous bounded holomorphic semigroup $\{S(z)\}$ on $X\left(\frac{\pi}{2}-\theta\right)$ such that $\|S(t)\| \leq 1$ in the real semi-axis $t \geq 0$. As $T(t)=e^{\beta t} S(t)$ for all $t \geq 0$, the result follows.

The arguments applied to $A$ can be reproduced on the infinitesimal generator $A^{*}$ of the adjoint semigroup $\left\{T(t)^{*}\right\}$ to obtain a symmetric operator $H_{*}$ on $(\mathfrak{H},\langle\cdot, \cdot\rangle)$ with $\mathcal{D}\left(H_{*}\right)=\mathcal{D}\left(A^{*}\right)$ such that

$$
\left\langle H_{*} u, v\right\rangle=\left\langle A^{*} u, v\right\rangle+\left\langle u, A^{*} v\right\rangle \quad \text { for all } u, v \in \mathcal{D}\left(A^{*}\right) .
$$

Lemma 3.2. Under the hypotheses of Lemma 3.1 one has that the symmetric operator $H_{*}$ in $(3.2)$ satisfies

$$
\left\langle H_{*} u, u\right\rangle \leq 2 \beta\|u\|^{2} \quad \text { for all } u \in \mathcal{D}\left(A^{*}\right)
$$


and

$$
\frac{d}{d t}\left\|T(t)^{*} h\right\|^{2}=\left\langle H_{*} T(t)^{*} h, T(t)^{*} h\right\rangle \quad \text { for all } t>0 \text { and } h \in \mathfrak{H} .
$$

Proof. Notice that $B^{*}\left(=(A-\beta)^{*}\right)$ is sectorial of the same angle $0<\theta<\frac{\pi}{2}$ as $B$ [8]. Besides, $B^{*}$ is $m$-dissipative, according to Proposition 2.1

We get two densely defined symmetric sesquilinear forms bounded from above with upper bound $2 \beta$ by setting

$$
x, y \in \mathcal{D}(A)=\mathcal{D}(H) \mapsto\langle H x, y\rangle \quad \text { and } \quad u, v \in \mathcal{D}\left(A^{*}\right)=\mathcal{D}\left(H_{*}\right) \mapsto\left\langle H_{*} u, v\right\rangle .
$$

Both forms are closable [9]. Their corresponding closures $\mathfrak{g}[\cdot, \cdot]$ and $\mathfrak{g}_{*}[\cdot, \cdot]$ are densely defined closed symmetric sesquilinear forms such that $\mathfrak{g}[x, x] \leq 2 \beta\|x\|^{2}$ for all $x \in \mathcal{D}(\mathfrak{g})$ and $\mathfrak{g}_{*}[u, u] \leq 2 \beta\|u\|^{2}$ for all $u \in \mathcal{D}\left(\mathfrak{g}_{*}\right)[9]$. The Friedrichs theorem for symmetric forms (cf. [9]) gives two selfadjoint operators $G, G_{*}$ which are bounded from above by $2 \beta$ and satisfy

$$
\frac{d}{d t}\|T(t) h\|^{2}=\langle G T(t) h, T(t) h\rangle, \quad \frac{d}{d t}\left\|T(t)^{*} h\right\|^{2}=\left\langle G_{*} T(t)^{*} h, T(t)^{*} h\right\rangle
$$
for all $t>0$ and $h \in \mathfrak{H}$.

A relevant fact established in [9] yields $G=G_{*}$.

Consider the polar decomposition $G=J|G|$, where $J$ is a selfadjoint partial isometry and $|G|$ is selfadjoint and nonnegative with $\mathcal{D}(|G|)=\mathcal{D}(G)$. Then write

$$
G x=G^{+} x+G^{-} x, \quad|G| x=G^{+} x-G^{-} x \quad(x \in \mathcal{D}(G)=\mathcal{D}(|G|)),
$$

where $G^{ \pm} \supseteq \frac{1}{2}(G \pm|G|)=\frac{1}{2}(J \pm 1)|G|$.

Lemma 3.3. The following assertions hold true:

(a) Every $x \in \mathcal{D}(G)$ satisfies $J x \in \mathcal{D}(G)$, GJx $=J G x=|G| x$, and $|G| J x=$ $J|G| x=G x$.

(b) $|\langle G x, x\rangle| \leq\langle|G| x, x\rangle$ for every $x \in \mathcal{D}(G)$.

(c) For all $x \in \mathcal{D}(G),\left\langle G^{-} x, x\right\rangle \leq 0$ and $0 \leq\left\langle G^{+} x, x\right\rangle \leq 2 \beta\|x\|^{2}$.

The proof is omitted.

Now we are ready to apply the Naimark-Arocena representation theorem to obtain a unitary dilation of $\{T(t)\}$. First we suppose that the infinitesimal generator $A$ is as in Lemma 3.1. To comply with the hypotheses of the theorem, take $\Gamma=\mathbb{R}$ and define $f: \mathbb{R} \rightarrow \mathfrak{L}(\mathfrak{H})$ by

$$
f(s):= \begin{cases}T(s), & \text { if } s \geq 0 \\ T(-s)^{*}, & \text { if } s<0\end{cases}
$$

It follows that $f$ is Hermitian and $f(0)=1$. Hence the kernel $(s, t) \mapsto f(s-t)$ is an operator-valued Toeplitz kernel on $\mathbb{R}$.

Let $h: \mathbb{R} \rightarrow \mathfrak{H}$ be a function with finite support. Write $\operatorname{supp}(h) \cup\{0\}=$ $\left\{\sigma_{j}\right\}_{j=0}^{Q} \cup\left\{\tau_{k}\right\}_{k=0}^{P}$, with $\sigma_{Q}<\cdots<\sigma_{1}<\sigma_{0}=0=\tau_{0}<\tau_{1}<\cdots<\tau_{P}$. Define 
$z\left(=z_{h}\right)$ and $y\left(=y_{h}\right)$ by $z(s):=\sum_{t \leq s} T(s-t)^{*} h(t) \quad(s \leq 0) \quad$ and $\quad y(s):=\sum_{t \geq s} T(t-s) h(t) \quad(s \geq 0)$.

Then

$$
h(s)= \begin{cases}z(s), & \text { if } s \leq \sigma_{Q} \\ z(s)-T\left(s-\sigma_{j}\right)^{*} z\left(\sigma_{j}\right), & \text { if } \sigma_{j}<s \leq \sigma_{j-1}, 1 \leq j \leq Q \\ y(s)-T\left(\tau_{k}-s\right) y\left(\tau_{k}\right), & \text { if } \tau_{k-1} \leq s<\tau_{k}, 1 \leq k \leq P \\ y(s), & \text { if } s \geq \tau_{P}\end{cases}
$$

In particular,

$$
z(0)-T\left(-\sigma_{1}\right)^{*} z\left(\sigma_{1}\right)=h(0)=y(0)-T\left(\tau_{1}\right) y\left(\tau_{1}\right)
$$

whence

$$
z(0)+T\left(\tau_{1}\right) y\left(\tau_{1}\right)=y(0)+T\left(-\sigma_{1}\right)^{*} z\left(\sigma_{1}\right) .
$$

If $v\left(=v_{h}\right)$ is given by

$$
v(s):= \begin{cases}z(s), & \text { if } s<0 \\ z(0)+T\left(\tau_{1}\right) y\left(\tau_{1}\right)=y(0)+T\left(-\sigma_{1}\right)^{*} z\left(\sigma_{1}\right), & \text { if } s=0 \\ y(s), & \text { if } s>0\end{cases}
$$

then, via the map $h \mapsto v$, we get that

$$
\begin{aligned}
\sum_{s, t \in \mathbb{R}}\langle f(s-t) h(s), h(t)\rangle \\
=\sum_{j=1}^{Q}\left\langle\left(1-T\left(\sigma_{j-1}-\sigma_{j}\right) T\left(\sigma_{j-1}-\sigma_{j}\right)^{*}\right) v\left(\sigma_{j}\right), v\left(\sigma_{j}\right)\right\rangle+\|v(0)\|^{2} \\
\quad+\sum_{k=1}^{P}\left\langle\left(1-T\left(\tau_{k}-\tau_{k-1}\right)^{*} T\left(\tau_{k}-\tau_{k-1}\right)\right) v\left(\tau_{k}\right), v\left(\tau_{k}\right)\right\rangle .
\end{aligned}
$$

Therefore, by 3.3,

$$
\begin{aligned}
\sum_{s, t \in \mathbb{R}}\langle f(s-t) h(s), h(t)\rangle & \\
= & \sum_{j=1}^{Q} \int_{\sigma_{j}}^{\sigma_{j-1}}\left\langle(-G) T\left(u-\sigma_{j}\right)^{*} v\left(\sigma_{j}\right), T\left(u-\sigma_{j}\right)^{*} v\left(\sigma_{j}\right)\right\rangle d u+\|v(0)\|^{2} \\
& +\sum_{k=1}^{P} \int_{\tau_{k-1}}^{\tau_{k}}\left\langle(-G) T\left(\tau_{k}-u\right) v\left(\tau_{k}\right), T\left(\tau_{k}-u\right) v\left(\tau_{k}\right)\right\rangle d u
\end{aligned}
$$


So, from Lemma 3.3 (b),

$$
\begin{aligned}
\left|\sum_{s, t \in \mathbb{R}}\langle f(s-t) h(s), h(t)\rangle\right| \\
\leq \sum_{j=1}^{Q} \int_{\sigma_{j}}^{\sigma_{j-1}}\left\langle|G| T\left(u-\sigma_{j}\right)^{*} v\left(\sigma_{j}\right), T\left(u-\sigma_{j}\right)^{*} v\left(\sigma_{j}\right)\right\rangle d u+\|v(0)\|^{2} \\
\quad+\sum_{k=1}^{P} \int_{\tau_{k-1}}^{\tau_{k}}\left\langle|G| T\left(\tau_{k}-u\right) v\left(\tau_{k}\right), T\left(\tau_{k}-u\right) v\left(\tau_{k}\right)\right\rangle d u \\
=\sum_{j=1}^{Q} \int_{\sigma_{j}}^{\sigma_{j-1}}\left\|C T\left(u-\sigma_{j}\right)^{*} v\left(\sigma_{j}\right)\right\|^{2} d u+\|v(0)\|^{2} \\
\quad+\sum_{k=1}^{P} \int_{\tau_{k-1}}^{\tau_{k}}\left\|C T\left(\tau_{k}-u\right) v\left(\tau_{k}\right)\right\|^{2} d u
\end{aligned}
$$

with $C:=|G|^{\frac{1}{2}}$. Straightforward computations give

$$
\begin{aligned}
\sum_{j=1}^{Q} \int_{\sigma_{j}}^{\sigma_{j-1}}\left\|C T\left(u-\sigma_{j}\right)^{*} v\left(\sigma_{j}\right)\right\|^{2} d u & \\
= & \sum_{s<0} \sum_{t<0}\left\langle\left(\int_{s \vee t}^{0} T(u-t)|G| T(u-s)^{*} d u\right) h(s), h(t)\right\rangle, \\
\|v(0)\|^{2}= & \sum_{s<0} \sum_{t<0}\left\langle T(-t) T(-s)^{*} h(s), h(t)\right\rangle+\sum_{s<0} \sum_{t \geq 0}\left\langle T(t-s)^{*} h(s), h(t)\right\rangle \\
& +\sum_{s \geq 0} \sum_{t<0}\langle T(s-t) h(s), h(t)\rangle+\sum_{s \geq 0} \sum_{t \geq 0}\left\langle T(t)^{*} T(s) h(s), h(t)\right\rangle
\end{aligned}
$$

and

$$
\begin{aligned}
& \sum_{k=1}^{P} \int_{\tau_{k-1}}^{\tau_{k}}\left\|C T\left(\tau_{k}-u\right) v\left(\tau_{k}\right)\right\|^{2} d u \\
& \quad=\sum_{s>0} \sum_{t>0}\left\langle\left(\int_{0}^{s \wedge t} T(t-u)^{*}|G| T(s-u) d u\right) h(s), h(t)\right\rangle .
\end{aligned}
$$

As a result, by setting

$$
k(s, t):= \begin{cases}\int_{s \vee t}^{0} T(u-t)|G| T(u-s)^{*} d u+T(-t) T(-s)^{*}, & s, t<0 \\ T(t-s)^{*}, & s<0, t \geq 0 \\ T(s-t), & s \geq 0, t<0 \\ T(t)^{*} T(s)+\int_{0}^{s \wedge t} T(t-u)^{*}|G| T(s-u) d u, & s \geq 0, t \geq 0\end{cases}
$$


we get an operator-valued kernel on $\mathbb{R}$ such that $k(0,0)=1$ and

$$
\left|\sum_{s, t \in \mathbb{R}}\langle f(s-t) h(s), h(t)\rangle\right| \leq \sum_{s, t \in \mathbb{R}}\langle k(s, t) h(s), h(t)\rangle
$$

for every $h: \mathbb{R} \rightarrow \mathfrak{H}$ with finite support.

It is worth noticing that, for $h: \mathbb{R} \rightarrow \mathfrak{H}$ with finite support and the corresponding $v: \mathbb{R} \rightarrow \mathfrak{H}$ as defined in 3.5 , we have that:

(1) If $\sigma_{j} \leq u<\sigma_{j-1}, 1 \leq j \leq Q$, then

$$
T\left(u-\sigma_{j}\right)^{*} v\left(\sigma_{j}\right)=\sum_{s \leq u} T(u-s)^{*} h(s)=v(u) .
$$

(2) If $\tau_{k-1}<u \leq \tau_{k}, 1 \leq k \leq P$, then

$$
T\left(\tau_{k}-u\right) v\left(\tau_{k}\right)=\sum_{s \geq u} T(s-u) h(s)=v(u) .
$$

Here, as before, $\sigma_{Q}<\cdots<\sigma_{1}<\sigma_{0}=0=\tau_{0}<\tau_{1}<\cdots<\tau_{P}$ are the real points in $\operatorname{supp}(h) \cup\{0\}$. Accordingly,

$$
\begin{aligned}
\sum_{s, t \in \mathbb{R}}\langle f(s-t) h(s), h(t)\rangle= & \sum_{j=1}^{Q} \int_{\sigma_{j}}^{\sigma_{j-1}}\langle(-G) v(u), v(u)\rangle d u+\|v(0)\|^{2} \\
& +\sum_{k=1}^{P} \int_{\tau_{k-1}}^{\tau_{k}}\langle(-G) v(u), v(u)\rangle d u
\end{aligned}
$$

and

$$
\begin{aligned}
\sum_{s, t \in \mathbb{R}}\langle k(s, t) h(s), h(t)\rangle= & \sum_{j=1}^{Q} \int_{\sigma_{j}}^{\sigma_{j-1}}\langle|G| v(u), v(u)\rangle d u+\|v(0)\|^{2} \\
& +\sum_{k=1}^{P} \int_{\tau_{k-1}}^{\tau_{k}}\langle|G| v(u), v(u)\rangle d u .
\end{aligned}
$$

If $\sum_{s, t \in \mathbb{R}}\langle k(s, t) h(s), h(t)\rangle>0$, set

$$
v^{\prime}(u):= \begin{cases}J v(u), & \text { if either } u<0 \text { or } u>0 \\ v(0), & \text { if } u=0 .\end{cases}
$$

A function $h^{\prime}: \mathbb{R} \rightarrow \mathfrak{H}$ with $\operatorname{supp}\left(h^{\prime}\right)=\operatorname{supp}(h)$ is uniquely determined by $v^{\prime}$ from (3.4. In fact, $h^{\prime}$ is given by

$$
h^{\prime}(s)= \begin{cases}v^{\prime}\left(\sigma_{Q}\right), & \text { if } s=\sigma_{Q} \\ v^{\prime}\left(\sigma_{j}\right)-T\left(\sigma_{j}-\sigma_{j+1}\right)^{*} v^{\prime}\left(\sigma_{j+1}\right), & \text { if } s=\sigma_{j}, 1 \leq j \leq Q-1 \\ v^{\prime}(0)-T\left(-\sigma_{1}\right)^{*} v^{\prime}\left(\sigma_{1}\right)-T\left(\tau_{1}\right) v^{\prime}\left(\tau_{1}\right), & \text { if } s=0 ; \\ v^{\prime}\left(\tau_{k}\right)-T\left(\tau_{k+1}-\tau_{k}\right) v^{\prime}\left(\tau_{k+1}\right), & \text { if } s=\tau_{k}, 1 \leq k \leq P-1 \\ v^{\prime}\left(\tau_{P}\right), & \text { if } s=\tau_{P} .\end{cases}
$$


Then the above discussion and Lemma 3.3 (a) yield

$$
\sum_{s, t \in \mathbb{R}}\left\langle f(s-t) h(s), h^{\prime}(t)\right\rangle=\sum_{s, t \in \mathbb{R}}\langle k(s, t) h(s), h(t)\rangle=\sum_{s, t \in \mathbb{R}}\left\langle k(s, t) h^{\prime}(s), h^{\prime}(t)\right\rangle .
$$

This shows that, for each $h: \mathbb{R} \rightarrow \mathfrak{H}$ supported on a finite set and satisfying that $\sum_{s, t \in \mathbb{R}}\langle k(s, t) h(s), h(t)\rangle>0$, there exists $h^{\prime}: \mathbb{R} \rightarrow \mathfrak{H}$ with finite support such that $\sum_{s, t \in \mathbb{R}}\left\langle k(s, t) h^{\prime}(s), h^{\prime}(t)\right\rangle>0$ and

$$
\frac{\left|\sum_{s, t \in \mathbb{R}}\left\langle f(s-t) h(s), h^{\prime}(t)\right\rangle\right|}{\left\{\sum_{s, t \in \mathbb{R}}\langle k(s, t) h(s), h(t)\rangle\right\}^{\frac{1}{2}}\left\{\sum_{s, t \in \mathbb{R}}\left\langle k(s, t) h^{\prime}(s), h^{\prime}(t)\right\rangle\right\}^{\frac{1}{2}}}=1 .
$$

It remains to verify that there exists a function $\rho: \mathbb{R} \rightarrow(0, \infty)$ such that

$$
\sum_{s, t \in \mathbb{R}}\langle k(s+\xi, t+\xi) h(s), h(t)\rangle \leq \rho(\xi) \sum_{s, t \in \mathbb{R}}\langle k(s, t) h(s), h(t)\rangle
$$

for all $h: \mathbb{R} \rightarrow \mathfrak{H}$ with finite support and all $\xi \in \mathbb{R}$.

Given $h: \mathbb{R} \rightarrow \mathfrak{H}$ with finite support, set

$$
h_{\xi}(s):=h(s-\xi) \quad(s \in \mathbb{R})
$$

and

$$
S(h, \xi):=\sum_{s, t \in \mathbb{R}}\langle k(s+\xi, t+\xi) h(s), h(t)\rangle
$$

so that

$$
S(h, \xi)=\sum_{s, t \in \mathbb{R}}\left\langle k(s, t) h_{\xi}(s), h_{\xi}(t)\right\rangle=S\left(h_{\xi}, 0\right) .
$$

As before, $\sigma_{Q}<\cdots<\sigma_{1}<\sigma_{0}=0=\tau_{0}<\tau_{1}<\cdots<\tau_{P}$ are the points in $\operatorname{supp}(h) \cup\{0\}$.

We first consider the case $\xi>0$.

The difference between the expressions for $S(h, \xi)$ and $S(h, 0)$ depends on the set $\operatorname{supp}(h) \cap[-\xi, 0)$. 
Particular case: $\sigma_{1} \leq-\xi<0$, i.e., $\operatorname{supp}(h) \cap[-\xi, 0)$ is either $\left\{\sigma_{1}\right\}\left(\sigma_{1}=-\xi\right)$ or $\emptyset\left(\sigma_{1}<-\xi\right)$. We obtain that

$$
\begin{aligned}
S(h, \xi)= & \sum_{j=1}^{Q} \int_{\sigma_{j}}^{\sigma_{j-1}}\left\langle|G| T\left(u-\sigma_{j}\right)^{*} v\left(\sigma_{j}\right), T\left(u-\sigma_{j}\right)^{*} v\left(\sigma_{j}\right)\right\rangle d u \\
& -\int_{0}^{\xi}\left\langle|G| T\left(u-\sigma_{1}-\xi\right)^{*} v\left(\sigma_{1}\right), T\left(u-\sigma_{1}-\xi\right)^{*} v\left(\sigma_{1}\right)\right\rangle d u \\
& +\left\|T\left(\sigma_{1}-\xi\right)^{*} v\left(\sigma_{1}\right)+T(\xi) y(0)\right\|^{2} \\
& +\int_{0}^{\xi}\langle|G| T(u-\xi) y(0), T(u-\xi) y(0)\rangle d u \\
& +\sum_{k=1}^{P} \int_{\tau_{k-1}}^{\tau_{k}}\left\langle|G| T\left(\tau_{k}-u\right) v\left(\tau_{k}\right), T\left(\tau_{k}-u\right) v\left(\tau_{k}\right)\right\rangle d u,
\end{aligned}
$$

where $y(0)=\sum_{t \geq 0} T(t) h(t)=v(0)-T\left(-\sigma_{1}\right)^{*} v\left(\sigma_{1}\right)$. Since $|G| x=2 G^{+} x-G x$ $(x \in \mathcal{D}(G))$, from 3.3 it follows that

$$
\begin{aligned}
S(h, \xi)= & S(h, 0)-2 \int_{0}^{\xi}\left\|\left(G^{+}\right)^{\frac{1}{2}} T\left(-t-\sigma_{1}\right)^{*} v\left(\sigma_{1}\right)\right\|^{2} d t \\
& +2 \int_{0}^{\xi}\left\|\left(G^{+}\right)^{\frac{1}{2}} T(t) y(0)\right\|^{2} d t \\
= & S(h, 0)-2 \int_{0}^{\xi}\left\|\left(G^{+}\right)^{\frac{1}{2}} T\left(-t-\sigma_{1}\right)^{*} v\left(\sigma_{1}\right)\right\|^{2} d t \\
& +2 \int_{0}^{\xi}\left\|\left(G^{+}\right)^{\frac{1}{2}} T(t)\left(v(0)-T\left(-\sigma_{1}\right)^{*} v\left(\sigma_{1}\right)\right)\right\|^{2} d t \\
\leq & S(h, 0)-2 \int_{0}^{\xi}\left\|\left(G^{+}\right)^{\frac{1}{2}} T\left(-t-\sigma_{1}\right)^{*} v\left(\sigma_{1}\right)\right\|^{2} d t \\
& +4 \int_{0}^{\xi}\left\|\left(G^{+}\right)^{\frac{1}{2}} T(t) v(0)\right\|^{2} d t \\
& +4 \int_{0}^{\xi}\left\|\left(G^{+}\right)^{\frac{1}{2}} T(t) T\left(-\sigma_{1}\right)^{*} v\left(\sigma_{1}\right)\right\|^{2} d t \\
\leq & S(h, 0)+4 \int_{0}^{\xi}\left\|\left(G^{+}\right)^{\frac{1}{2}} T(t) v(0)\right\|^{2} d t \\
& +4 \int_{0}^{\xi}\left\|\left(G^{+}\right)^{\frac{1}{2}} T(t) T\left(-\sigma_{1}\right)^{*} v\left(\sigma_{1}\right)\right\|^{2} d t .
\end{aligned}
$$

We apply Lemma 3.3 (c) and get

$$
4 \int_{0}^{\xi}\left\|\left(G^{+}\right)^{\frac{1}{2}} T(t) v(0)\right\|^{2} d t \leq 8 \beta \int_{0}^{\xi}\|T(t) v(0)\|^{2} d t \leq 8 \beta\left(\int_{0}^{\xi} e^{2 \beta t} d t\right)\|v(0)\|^{2}
$$


and

$$
\begin{aligned}
4 \int_{0}^{\xi}\left\|\left(G^{+}\right)^{\frac{1}{2}} T(t) T\left(-\sigma_{1}\right)^{*} v\left(\sigma_{1}\right)\right\|^{2} d t \\
=4 \int_{0}^{\xi}\left\|\left(G^{+}\right)^{\frac{1}{2}} T(t) T(t)^{*} T\left(-t-\sigma_{1}\right)^{*} v\left(\sigma_{1}\right)\right\|^{2} d t \\
\leq 8 \beta \int_{0}^{\xi} e^{2 \beta t}\left\|T(t)^{*} T\left(-t-\sigma_{1}\right)^{*} v\left(\sigma_{1}\right)\right\|^{2} d t \\
=8 \beta \int_{0}^{\xi} \int_{0}^{t} e^{2 \beta t}\left\langle G T(s)^{*} T\left(-t-\sigma_{1}\right)^{*} v\left(\sigma_{1}\right), T(s)^{*} T\left(-t-\sigma_{1}\right)^{*} v\left(\sigma_{1}\right)\right\rangle d s d t \\
\quad-8 \beta \int_{0}^{\xi} e^{2 \beta t}\left\|T\left(-t-\sigma_{1}\right)^{*} v\left(\sigma_{1}\right)\right\|^{2} d t \\
\leq 8 \beta \int_{0}^{\xi} \int_{0}^{t} e^{2 \beta t}\left\langle G T(s)^{*} T\left(-t-\sigma_{1}\right)^{*} v\left(\sigma_{1}\right), T(s)^{*} T\left(-t-\sigma_{1}\right)^{*} v\left(\sigma_{1}\right)\right\rangle d s d t \\
=8 \beta \int_{0}^{\xi} \int_{0}^{t} e^{2 \beta t}\left\langle G T\left(s-t-\sigma_{1}\right)^{*} v\left(\sigma_{1}\right), T\left(s-t-\sigma_{1}\right)^{*} v\left(\sigma_{1}\right)\right\rangle d s d t \\
=8 \beta \int_{0}^{\xi} \int_{0}^{x} e^{2 \beta x}\left\langle G T\left(-y-\sigma_{1}\right)^{*} v\left(\sigma_{1}\right), T\left(-y-\sigma_{1}\right)^{*} v\left(\sigma_{1}\right)\right\rangle d y d x \\
\leq 8 \beta\left(\int_{0}^{\xi} e^{2 \beta x} d x\right)\left(\int_{0}^{\xi}\left\|C T\left(-y-\sigma_{1}\right)^{*} v\left(\sigma_{1}\right)\right\|^{2} d y\right) \\
=8 \beta\left(\int_{0}^{\xi} e^{2 \beta x} d x\right)\left(\int_{-\xi}^{0}\left\|C T\left(u-\sigma_{1}\right)^{*} v\left(\sigma_{1}\right)\right\|^{2} d u\right) .
\end{aligned}
$$

It thereby follows that

$$
S(h, \xi)-S(h, 0) \leq 8 \beta\left(\int_{0}^{\xi} e^{2 \beta t} d t\right)\left(\|v(0)\|^{2}+\int_{-\xi}^{0}\left\|C T\left(u-\sigma_{1}\right)^{*} v\left(\sigma_{1}\right)\right\|^{2} d u\right) .
$$

Since

$$
8 \beta \int_{0}^{\xi} e^{2 \beta t} d t \leq 8 \beta \int_{0}^{\xi} e^{8 \beta t} d t=e^{8 \beta \xi}-1
$$

and

$$
\|v(0)\|^{2}+\int_{-\xi}^{0}\left\|C T\left(u-\sigma_{1}\right)^{*} v\left(\sigma_{1}\right)\right\|^{2} d u \leq S(h, 0)
$$

it follows that

$$
S(h, \xi) \leq e^{8 \beta \xi} S(h, 0) .
$$

We already mentioned that the cases to be considered are linked up with the points in $\operatorname{supp}(h) \cap[-\xi, 0)$. Next we will see that all cases can be reduced to the particular one.

If $\sigma_{2} \leq-\xi<\sigma_{1}$ then $S(h, \xi)=S\left(h,\left(\xi+\sigma_{1}\right)-\sigma_{1}\right)=S\left(h_{-\sigma_{1}}, \xi+\sigma_{1}\right)$, where $\sigma_{2}-\sigma_{1} \leq-\left(\xi+\sigma_{1}\right)<0$, which is to say that $\operatorname{supp}\left(h_{-\sigma_{1}}\right) \cap\left[-\left(\xi+\sigma_{1}\right), 0\right)$ is either 
$\left\{\sigma_{2}-\sigma_{1}\right\}\left(\sigma_{2}=-\xi\right)$ or $\emptyset\left(\sigma_{2}<-\xi\right)$. Therefore, $h_{-\sigma_{1}}$ and $\xi+\sigma_{1}$ in the sum $S\left(h_{-\sigma_{1}}, \xi+\sigma_{1}\right)$ are like in the particular case, whence

$$
S\left(h_{-\sigma_{1}}, \xi+\sigma_{1}\right) \leq e^{8 \beta\left(\xi+\sigma_{1}\right)} S\left(h_{-\sigma_{1}}, 0\right)=e^{8 \beta\left(\xi+\sigma_{1}\right)} S\left(h,-\sigma_{1}\right) .
$$

Since $h$ and $-\sigma_{1}$ in the sum $S\left(h,-\sigma_{1}\right)$ accord with the particular case as well, we get $S\left(h,-\sigma_{1}\right) \leq e^{-8 \beta \sigma_{1}} S(h, 0)$, hence 3.7$)$. The case $\sigma_{3} \leq-\xi<\sigma_{2}$ can be carried over into the previous one and so on.

The case $\xi<0$ can be handled in a similar way. We get that

$$
S(h, \xi) \leq e^{-8 \beta \xi} S(h, 0) .
$$

Therefore (3.6) holds with $\rho(\xi):=e^{8 \beta|\xi|}$.

We have proved the following result.

Theorem 3.4. Let $\{T(t)\}$ be a strongly continuous semigroup of bounded linear operators on a Hilbert space $(\mathfrak{H},\langle\cdot, \cdot\rangle)$. Let $A$ be the infinitesimal generator of $\{T(t)\}$. Suppose that there exists $\beta \geq 0$ such that $A-\beta$ is sectorial of angle $0<\theta<\frac{\pi}{2}$ and $\operatorname{Re}\langle A x, x\rangle \leq \beta\|x\|^{2}$ for all $x \in \mathcal{D}(A)$. Then there exist a Krein space $\left(\mathfrak{K},\langle\cdot, \cdot\rangle_{\mathfrak{K}}\right)$ containing $(\mathfrak{H},\langle\cdot, \cdot\rangle)$ as a regular subspace and a strongly continuous unitary group $\{U(t)\} \subseteq \mathfrak{L}(\mathfrak{K})$ such that:

(a) $T(t)=\left.P_{\mathfrak{H}} U(t)\right|_{\mathfrak{H}}$ and $T(t)^{*}=\left.P_{\mathfrak{H}} U(-t)\right|_{\mathfrak{H}}$ for all $t \geq 0$ (dilation property);

(b) $\bigvee\{U(t) \mathfrak{H}: t \in \mathbb{R}\}$ is dense in $\mathfrak{K}$ (minimality condition).

It is all set for the result we announced in the Introduction.

Theorem 3.5. Let $\{T(t)\}$ be a strongly continuous semigroup of bounded linear operators on a Hilbert space $(\mathfrak{H},\langle\cdot, \cdot\rangle)$. Let $A$ be the infinitesimal generator of $\{T(t)\}$. Suppose that there exists $\beta \geq 0$ such that $A-\beta$ is sectorial of angle $0<\theta<\frac{\pi}{2}$ and $\operatorname{Re}\langle A x, x\rangle_{0} \leq \beta\|x\|_{0}^{2}$ for all $x \in \mathcal{D}(A)$, with $\langle\cdot, \cdot\rangle_{0}$ an equivalent scalar product on $\mathfrak{H}$. Then there exist a Krein space $\left(\mathfrak{K},\langle\cdot, \cdot\rangle_{\mathfrak{K}}\right)$ containing $(\mathfrak{H},\langle\cdot, \cdot\rangle)$ as a regular subspace and a strongly continuous unitary group $\{U(t)\} \subseteq \mathfrak{L}(\mathfrak{K})$ such that:

(a) $T(t)=\left.P_{\mathfrak{H}} U(t)\right|_{\mathfrak{H}}$ and $T(t)^{*}=\left.P_{\mathfrak{H}} U(-t)\right|_{\mathfrak{H}}$ for all $t \geq 0$.

(b) $\bigvee\{U(t) \mathfrak{H}: t \in \mathbb{R}\}$ is dense in $\mathfrak{K}$.

Proof. By Theorem 3.4 we already have a Kreŭn space $\left(\mathfrak{K}_{0},\langle\cdot, \cdot\rangle_{\mathfrak{K}_{0}}\right)$ containing $\left(\mathfrak{H},\langle\cdot, \cdot\rangle_{0}\right)$ as a regular subspace and a strongly continuous unitary group $\left\{U_{0}(t)\right\} \subseteq$ $\mathfrak{L}\left(\mathfrak{K}_{0}\right)$ such that $\left(\mathfrak{K}_{0},\left\{U_{0}(t)\right\}\right)$ is a minimal unitary dilation of $\{T(t)\} \subseteq \mathfrak{L}(\mathfrak{H})$.

We can choose a fundamental decomposition of $\mathfrak{K}_{0}$ such that $\mathfrak{H} \subseteq \mathfrak{K}_{0}^{+}$, say $\mathfrak{K}_{0}=\left(\mathfrak{H} \oplus \mathfrak{M}^{+}\right) \oplus \mathfrak{K}_{0}^{-}$. Consider the linear space $\mathfrak{K}:=\mathfrak{K}_{0}$ with the scalar product

$$
\left\langle h+b, h^{\prime}+b^{\prime}\right\rangle_{\mathfrak{K}}:=\left\langle h, h^{\prime}\right\rangle+\left\langle b, b^{\prime}\right\rangle_{\mathfrak{K}_{0}} \quad\left(h, h^{\prime} \in \mathfrak{H}, b, b^{\prime} \in \mathfrak{M}^{+} \oplus \mathfrak{K}_{0}^{-}\right) .
$$

It is clear that $\left(\mathfrak{K},\langle\cdot, \cdot\rangle_{\mathfrak{K}}\right)$ is a Krel̆n space containing $(\mathfrak{H},\langle\cdot, \cdot\rangle)$ as a regular subspace for a fundamental decomposition can be chosen to be

$$
\mathfrak{K}=\left(\mathfrak{H} \oplus \mathfrak{M}^{+}\right) \oplus \mathfrak{K}_{0}^{-} .
$$

The corresponding Hilbert space scalar product is

$$
\left\langle h+b, h^{\prime}+b^{\prime}\right\rangle_{|\mathfrak{K}|}:=\left\langle h, h^{\prime}\right\rangle+\left\langle b, b^{\prime}\right\rangle_{\left|\mathfrak{K}_{0}\right|} \quad\left(h, h^{\prime} \in \mathfrak{H}, b, b^{\prime} \in \mathfrak{M}^{+} \oplus \mathfrak{K}_{0}^{-}\right) .
$$


There exist $d, D>0$ such that $d\|h\|_{0} \leq\|h\| \leq D\|h\|_{0}$ for all $h \in \mathfrak{H}$. Thus, there exists a linear operator $\Lambda$ on $\mathfrak{H}$ such that

$$
\left\langle h, h^{\prime}\right\rangle=\left\langle\Lambda h, h^{\prime}\right\rangle_{0} \quad \text { for all } h, h^{\prime} \in \mathfrak{H}
$$

and

$$
d^{2}\|h\|_{0}^{2} \leq\langle\Lambda h, h\rangle_{0} \leq D^{2}\|h\|_{0}^{2} \quad \text { for all } h \in \mathfrak{H} .
$$

Besides, $\Lambda$ is $\langle\cdot, \cdot\rangle$-positive (hence, bounded in the norm $\|\cdot\|$ ). Therefore $\Lambda$ is positive, bounded and boundedly invertible in both Hilbert spaces $\left(\mathfrak{H},\langle\cdot, \cdot\rangle_{0}\right)$ and $(\mathfrak{H},\langle\cdot, \cdot\rangle) . \Lambda$ can be extended to a linear operator $\widetilde{\Lambda}$ on all of $\mathfrak{K}=\mathfrak{K}_{0}$ by putting $\widetilde{\Lambda} h:=\Lambda h(h \in \mathfrak{H})$ and $\widetilde{\Lambda} b:=b\left(b \in \mathfrak{M}^{+} \oplus \mathfrak{K}_{0}^{-}\right)$. It follows that

$$
\left\langle k, k^{\prime}\right\rangle_{\mathfrak{K}}=\left\langle\widetilde{\Lambda} k, k^{\prime}\right\rangle_{\mathfrak{K}_{0}}=\left\langle k, \widetilde{\Lambda} k^{\prime}\right\rangle_{\mathfrak{K}_{0}} \quad \text { for all } k, k^{\prime} \in \mathfrak{K}=\mathfrak{K}_{0}
$$

and

$$
\left(d^{2} \wedge 1\right)\|k\|_{\left|\mathfrak{K}_{0}\right|}^{2} \leq\langle\widetilde{\Lambda} k, k\rangle_{\left|\mathfrak{K}_{0}\right|} \leq\left(D^{2} \vee 1\right)\|k\|_{\left|\mathfrak{K}_{0}\right|}^{2} \text { for all } k \in \mathfrak{K}_{0} .
$$

Also, with respect to the decomposition $\mathfrak{K}=\mathfrak{K}_{0}=\mathfrak{H} \oplus\left(\mathfrak{M}^{+} \oplus \mathfrak{K}_{0}^{-}\right), \widetilde{\Lambda}$ can be represented in the form

$$
\widetilde{\Lambda}=\left[\begin{array}{cc}
\Lambda & 0 \\
0 & 1
\end{array}\right]=\left[\begin{array}{cc}
\Lambda^{\frac{1}{2}} & 0 \\
0 & 1
\end{array}\right]\left[\begin{array}{cc}
\Lambda^{\frac{1}{2}} & 0 \\
0 & 1
\end{array}\right]
$$

Thus, if $L:=\left[\begin{array}{cc}\Lambda^{\frac{1}{2}} & 0 \\ 0 & 1\end{array}\right]$ then $L$ is positive, bounded and boundedly invertible in both Krĕn spaces $\left(\mathfrak{K},\langle\cdot, \cdot\rangle_{\mathfrak{K}}\right)$ and $\left(\mathfrak{K}_{0},\langle\cdot, \cdot\rangle_{\mathfrak{K}_{0}}\right)$.

Define $U(t):=L^{-1} U_{0}(t) L(t \in \mathbb{R})$. It can be seen that $\{U(t)\} \subseteq \mathfrak{L}(\mathfrak{K})$ is a strongly continuous unitary group with the desired properties.

\section{REFERENCES}

[1] T. Ando, Linear operators on Krĕ̌n spaces, Hokkaido University, Research Institute of Applied Electricity, Division of Applied Mathematics, Sapporo, 1979. MR 0560903

[2] R. Arocena, Scattering functions, Fourier transforms of measures, realization of linear systems and dilations of operators to Krel̆ spaces: a unified approach, in Seminar on harmonic analysis, 1983-1984, 1-55, Publ. Math. Orsay, 85-2, Univ. Paris XI, Orsay, 1985. MR 0802776

[3] T. Ya. Azizov and I. S. Iokhvidov, Linear operators in spaces with an indefinite metric, Wiley, Chichester, 1989. MR 1033489

[4] J. Bognár, Indefinite inner product spaces, Springer-Verlag, New York, 1974. MR 0467261.

[5] C. Davis, Dilation of uniformly continuous semi-groups, Rev. Roumaine Math. Pures Appl. 15 (1970), 975-983. MR 0268718.

[6] M. A. Dritschel and J. Rovnyak, Extension theorems for contraction operators on Kre ̌n spaces, in Extension and interpolation of linear operators and matrix functions, 221-305, Oper. Theory Adv. Appl., 47, Birkhäuser, Basel, 1990. MR 1120277

[7] M. A. Dritschel and J. Rovnyak, Operators on indefinite inner product spaces, in Lectures on operator theory and its applications (Waterloo, ON, 1994), 141-232, Fields Inst. Monogr., 3, Amer. Math. Soc., Providence, RI. MR 1364446.

[8] M. Haase, The functional calculus for sectorial operators, Operator Theory: Advances and Applications, 169, Birkhäuser Verlag, Basel, 2006. MR 2244037

[9] T. Kato, Perturbation theory for linear operators, Springer-Verlag, Berlin, 1980.

[10] B. McEnnis, Dilations of holomorphic semigroups, J. Operator Theory 23 (1990), no. 1, 21-42. MR 1054813 
[11] A. Pazy, Semigroups of linear operators and applications to partial differential equations, Applied Mathematical Sciences, 44, Springer-Verlag, New York, 1983. MR 0710486

[12] K. Yosida, Functional analysis, fourth edition, Springer-Verlag, New York, 1974. MR 0350358

Stefania A. M. Marcantognini

Department of Mathematics, Instituto Venezolano de Investigaciones Científicas, Km. 11

Carretera Panamericana, Altos de Pipe, Edo. Miranda, Venezuela;

CONICET - Instituto Argentino de Matemática "Alberto P. Calderón”, Saavedra 15, Piso 3, C1083 CABA, Argentina; and

Instituto de Ciencias, Universidad Nacional de General Sarmiento, Juan María Gutiérrez 1150, B1613 Los Polvorines, Pcia. de Buenos Aires, Argentina

stefania.marcantognini@gmail.com

Received: November 15, 2018

Accepted: April 23, 2019 